\title{
Impact of Water Content on the Electrical Behavior of Epoxy Insulators
}

\author{
E. Mboungou, C. Mavon, J.-M. Friedt, C. Bergeon and M. Fromm \\ Laboratoire de Microanalyses Nucléaires Alain Chambaudet (LMN-AC), \\ UMR-CEA E4, Université de Franche-Comté, UFR des Sciences et Techniques, \\ 16 route de Gray, 25030 Besançon cedex, France
}

\begin{abstract}
Polymers are widely used in indoor insulation. We analyze three epoxy samples with varying matrix and fillerproperties to determine the influence of water on their insulating properties. We observed a strong dependency of the leakage current and the sample temperature, under electrical stress, on the amount of absorbed water. Trihydrated alumina filler reduced the leakage current and temperature rise under electrical stress.
\end{abstract}

Index Terms — Electrical tests, epoxy resin, Diglycidyl Ether Bisphenol A resin (DGEBA), tri-hydrated alumina.

\section{INTRODUCTION}

INSULATORS in high-voltage devices are critical components since a breakdown of the insulation can lead to catastrophic damage in the electrical network. Therefore, the life time of insulation in devices is required to be typically 30 years. The insulating properties of the materials must be considered as a combination of their physical parameters and their response during aging to temperature, humidity and mechanical stress.

Composite materials are widely used in this area, both for bulk and surface insulation. However, the long term stability of these materials often remains a point of concern in their application and hence deserves further investigation.

Usually, insulating materials are chosen on the basis of the desired electrical, mechanical and chemical properties. For example, silicone rubbers are commonly used in outdoor insulation applications because of their good aging behavior, which is attributed to the hydrophobicity of the surface, restored in time by a mechanism involving the migration of light molecules from the bulk towards the surface [1-3]. For these reasons, accelerated aging experiments are necessary:

- to understand aging mechanisms and the influence of several stresses on the degradation of the insulation,

- to propose alternative materials with better properties and/or lower costs.

Aging of insulators may be accelerated by large changes in temperature and humidity [4], voltage [5] or corona effect of water drops [6-8]. The materials under investigation here are used in indoor devices and are not submitted to outdoor stresses like rain, ice or dust. However, temperature variations can occur as well as variations of humidity. In some cases, these two effects can lead to condensation on the surface of the insulator. It has been reported that early aging of the insulator surface occurs when the devices are submitted to wide variations of relative humidity (from $50 \%$ RH up to $98 \%$ $\mathrm{RH})$. Thus, an appropriate way to accelerate aging is to submit the insulators to extended cycles of humidity.

The common description of degradation is known as the "dry-bands" mechanism, which induces surface erosion and cracks [9]. Before breakdown, the aging process is described by two main steps. The first one is called the "early aging period" (EAP) during which the leakage current is weak and capacitive, and partial discharges occur occasionally. At the end of the EAP, partial discharge rate increases and makes the surface less hydrophobic. The surface of the insulator becomes wet. Then, the "late aging period" (LAP) sets in, characterized by an increase of the leakage current with a predominantly resistive component $[1,7,10]$.

The present study is part of a global research program, aiming at the determination of the influence of intrinsic properties (hydrophobicity, absorbance of water, hydrolysis of the matrix) on the degradation kinetics of some composite insulators of practical interest. Emphasis is placed on the understanding and improvement of the initial steps of the degradation, with the goal of improving the life time of the insulators and guarantee long term (30 years) insulating quality. The results discussed in this paper are obtained with 3 composites including the same matrix: a Diglycidyl Ether Bisphenol A resin (DGEBA)-based epoxy resin. The comparison of the results will emphasize the influence of two parameters:

- the absorption of water,

- the nature of the filler.

2 EXPERIMENTAL SETUP, MATERIALS AND PROCEDURE 


\subsection{EXPERIMENTAL SETUP}

The experimental set-up consists of three main parts: the climatic chamber defines the environmental conditions (temperature and moisture level) around the sample, the test chamber includes all parts exposed to high-voltages within a Faraday cage, and the data acquisition setup controls the environmental conditions around the sample while acquiring data representative of the environment and the electrical behavior of the sample under investigation.

\subsubsection{THE CLIMATIC CHAMBER}

Simulating operating conditions of the materials under test requires a constant humidity source. A Binder KBF 115 climatic chamber provides air at constant temperature and humidity with an accuracy after calibration of $\pm 3 \%$.

\subsubsection{THE TEST CHAMBER}

Samples are submitted to electrical and environmental stresses within the test chamber, which is made of a $30 \mathrm{~cm} \times 30$ $\mathrm{cm} \times 30 \mathrm{~cm}$ poly(methyl methacrylate) (PMMA) box, located in a Faraday cage. This chamber is connected to the climatic chamber by two tubings, hence generating an air flow. The actual humidity level in our test chamber is monitored by a Honeywell HIH-3610 humidity sensor which provides a readout accurate to $\pm 2 \%$ after calibration. The moisture level within the test chamber is regulated by a motorized valve controlled by a lab-made software PID leading to typical rise and fall durations between 40 and $98 \%$ relative humidity $(\mathrm{RH})$ of about 2 minutes. A pair of electrodes has been designed to avoid tip effects and provide a quasi-homogeneous electric field in the central part of the sample. In order to reduce the degradation effects induced by charge injection at a metalinsulator interface [11] and the formation of whiskers due to fusion of the metallic electrodes, the contact between electrodes and the sample is made through a conducting carbon loaded elastomer. The average electric field along the sample is $0.45 \mathrm{kV} / \mathrm{mm}$. The applied voltage is provided by a 600 VA step-up transformer providing up to $20 \mathrm{kV}$, which is supplied by a variable autotransformer. The actual applied voltage is measured by a high impedance probe with $1 / 1000$ gain. The leakage current through the sample is monitored as a voltage drop through a $1000 \Omega$ resistor. Finally, a $100 \mu \mathrm{m}$ diameter type $\mathrm{K}$ thermocouple, monitored via an AD595 monolithic thermocouple amplifier with cold junction compensation, is placed in a $0.8 \mathrm{~mm}$ diameter hole drilled in the side of the sample for measurement of the temperature inside the sample during electrical tests.

\subsubsection{DATA ACQUISITION PROCEDURE}

All experimental parameters are monitored and recorded through a 16 channel, 12-bit, $250 \mathrm{k}$ samples/s analog to digital converter as provided by a National Instrument PCI-6040E card. The control software was written under LabView (National Instruments), and records once every second the 50 $\mathrm{Hz}$ component of the current, its phase with respect to the applied voltage, test cell temperature and humidity, sample temperature, and applied voltage magnitude.

\subsection{MATERIALS}

All materials involved in this study are based on DGEBA resin as used in low and medium voltage equipments. This resin is synthesized from 2,2-bis(4'-hydroxylphenyl)propane also named bisphenol A, and 1-chloro-2,3-epoxyde propane also named epichlorhydrine. Figure 1 presents the chemical formula of DGEBA.

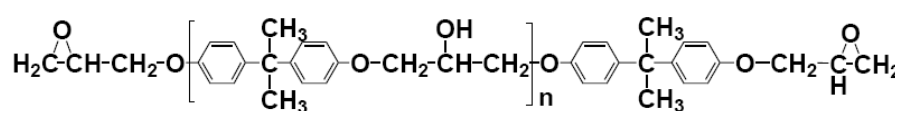

Figure 1. Structure of DGEBA resin.

This resin is processed with an hardener and an accelerator in order to produce a reticulated solid. Differential Scanning Calorimetry (DSC, Figure 2) shows different glass transition temperatures $\mathrm{T}_{\mathrm{g}}$ for one sample compared to the other two: we will from now on refer to this first sample as a rough silicaloaded sample (as identified by the surface properties of the mold used in this case: $\mathrm{T}_{\mathrm{g}} \sim 70{ }^{\circ} \mathrm{C}$ ), while the other two samples displaying higher glass transition temperatures $(\mathrm{Tg} \sim$ $124^{\circ} \mathrm{C}$ ) will be referred to as smooth silica-loaded epoxy and (smooth) THA-loaded epoxy. These samples were obtained with different processes leading to different reticulation properties and hence different $\mathrm{T}_{\mathrm{g}}$.

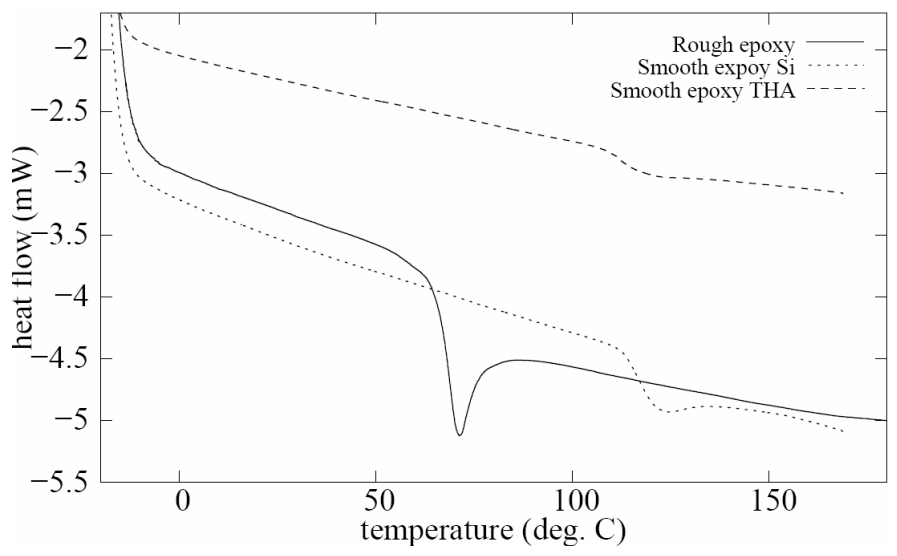

Figure 2. Differential Scanning Calorimetry scan (increasing temperature) of the three samples under investigation: the glass transition is visible as a heat flow drop.

In order to improve the mechanical characteristics of these products, fillers are added to the resin. In this study, two types of fillers are used: silica $(\mathrm{SiO} 2)$ which is the filler used by our industrial partner during the fabrication of its electrical equipment : and tri-hydrated alumina (THA), $\left(\mathrm{Al}_{2} \mathrm{O}_{3}, 3 \mathrm{H}_{2} \mathrm{O}\right)$.

The final mixture is molded in plates which are cut and cleaned to provide our test samples. Two kinds of molds have been used: one with a rougher surface hence resulting in rough samples, and the other mirror polished leading to smooth samples. Table 1 summarizes the properties of these materials.

\subsection{EXPERIMENTAL PROTOCOLS}

Each sample, after being cut from the original plate, is conditioned either under vacuum or soaked in water. In both 
Table 1. Summary of the characteristics of the epoxy-based materials used in this study.

\begin{tabular}{c|c|c}
\hline Samples & Nature of the surface & Nature of the filler \\
\hline Rough silica-filled \\
epoxy \\
$\begin{array}{c}\text { Rough silica-filled } \\
\text { epoxy } \\
\text { Smooth THA-filled } \\
\text { epoxy }\end{array}$ & $\begin{array}{c}\text { Rough and } \\
\text { hydrophobic } \\
\text { Rough and } \\
\text { hydrophobic } \\
\text { Smooth and } \\
\text { hydrophobic }\end{array}$ & Silica \\
\hline
\end{tabular}

cases, the sample weight is measured daily to estimate water loss and absorption respectively. Each sample is then submitted to an electrical test: Figure 3 presents a picture of the sample holder used for applying the electric field, parallel to the sample, between the two upper electrodes. Each sample is clamped on both sides, the upper side in which the electrodes are brought closest (spacing: $3.5 \mathrm{~cm}$ ) being submitted to the most significant electrical field.

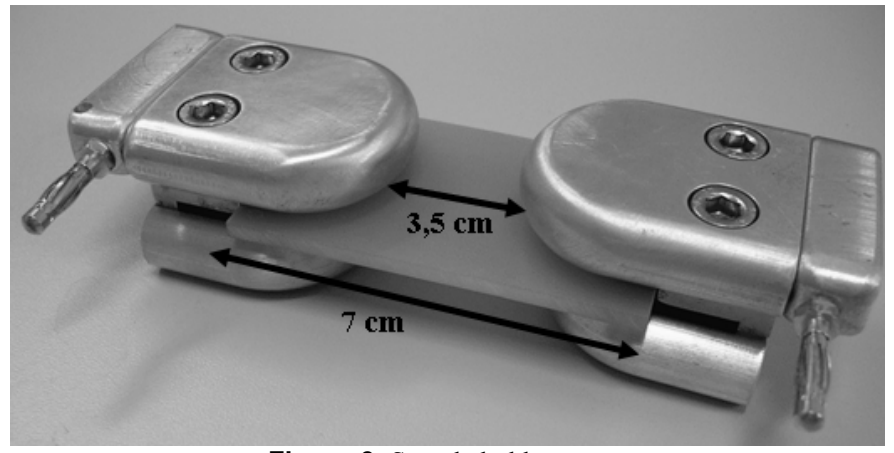

Figure 3. Sample holder

Electrical tests are performed by applying a $15.75 \mathrm{kVrms}$ $\mathrm{AC}$ voltage for 8 hours, leading to a mean electric field of 0.45 $\mathrm{kVrms} / \mathrm{mm}$ between the electrodes. During the whole electrical test, the ambient humidity level is cycled between a wet condition in which the chamber is kept at a relative humidity (RH) level of $98 \%$, and a dry phase during which the RH is kept at $40 \%$. The temperature of the test chamber is kept constant at $40 \pm 2{ }^{\circ} \mathrm{C}$. During the $8 \mathrm{~h}$ of the test, all parameters mentioned earlier (section 2.1.3) are continuously recorded.

\section{RESULtS \\ 3.1 SAMPLE ROUGNESS}

Data on the surface roughness of all samples considered in this study were recorded using a tactile profiler fitted with a $2.52 \mu \mathrm{m}$ curvature radius diamond tip probe (Digital Surf, Besanc, on, France). Topography data were processed using the software Mountain Map Universal (v.3.1.0). The theoretical sampling resolution in both in plane directions is 1

Table 2. Rougness parameters of the surfaces: $S_{a}$ is the roughness average, $\mathrm{S}_{\mathrm{q}}$ is the root mean square of the roughness in $\mu \mathrm{m}$.

\begin{tabular}{ccc|c|c}
\hline $\begin{array}{c}\text { Roughness } \\
\text { parameter }\end{array}$ & $\begin{array}{c}\text { Rough } \\
\text { silica-filled } \\
\text { epoxy }\end{array}$ & $\begin{array}{c}\text { Smooth } \\
\text { silica-filled } \\
\text { epoxy }\end{array}$ & $\begin{array}{c}\text { Smooth } \\
\text { THA-filled } \\
\text { epoxy }\end{array}$ & $\begin{array}{c}\text { Rough } \\
\text { silica-filled } \\
\text { epoxy } \\
\text { Polished }\end{array}$ \\
\hline $\mathrm{S}_{\mathrm{a}}(\mu \mathrm{m})$ & 0.978 & 0.136 & 0.186 & 0.122 \\
\hline $\mathrm{S}_{\mathrm{q}}(\mu \mathrm{m})$ & 1.25 & 0.209 & 0.242 & 0.194 \\
\hline
\end{tabular}

$\mu \mathrm{m}$. Table 2 summarizes the roughness parameters extracted from these scans for the three raw samples.

In addition to differences in the surface roughness arising from different roughnesses of the mold, DSC analysis shows that the resins used for preparing the two silica-loaded epoxy (Si-epoxy) samples were processed differently. Hence chemical composition will be of interest, in addition to surface roughness. The comparison of the behavior between smooth and rough Si-epoxy is thus representative of the variability of the characteristics of the resins as a function of production methods. Reproducibility is however excellent within each sample set obtained following a given procedure.

\subsection{WATER SORPTION}

Figure 4 presents the water absorption levels as a function of time for epoxy samples: rough sample, smooth samples with $\mathrm{Si}$ or THA fillers, as well as a rough sample polished in order to achieve a surface roughness similar to that of the smooth samples. The first observation is that the rough silica-loaded sample absorbs approximately twice as much water as the smooth sample. On the other hand, we observe no difference in the amount of absorbed water between the raw rough sample and the polished rough sample. Finally, the amount of water absorbed by the THA-loaded epoxy is slightly larger than that of the smooth silica loaded sample, but lower than that of the rough epoxy with or without polishing.

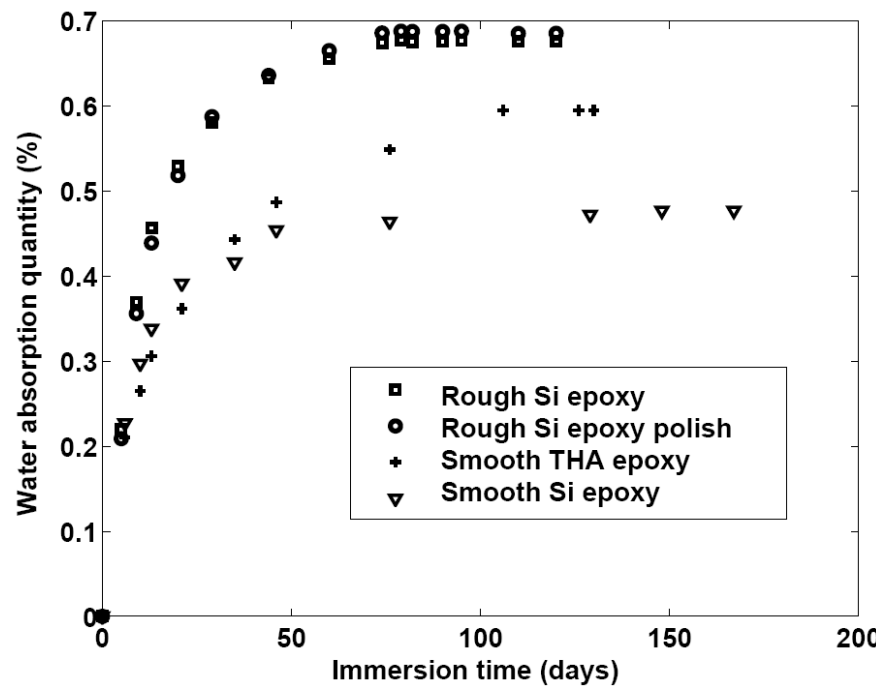

Figure 4. Water absorption measurements as a function of time for epoxy samples.

\subsection{ELECTRICAL RESPONSES 3.3.1 ELECTRICAL RESPONSES OF THA-FILLED SMOOTH EPOXY}

Figure 5 presents the evolution of the leakage current as a function of time and absorbed water content. Leakage current variations are strongly correlated to humidity level variations. We observe an increase of the leakage current when reaching the wet part of the humidity cycle and a decrease during the dry phase. The variations are strongest for large water absorption levels: 0.50 and $0.58 \%$. The initial leakage current slightly increases with increasing absorbed water amount, with mean values measured at $7.0 \pm 0.1,7.5 \pm 0.1,7.5 \pm 0.1,8.0 \pm 0.1$ 
and $9.0 \pm 0.1 \mu \mathrm{A}$ when increasing the water absorption levels as show in Figure 5.

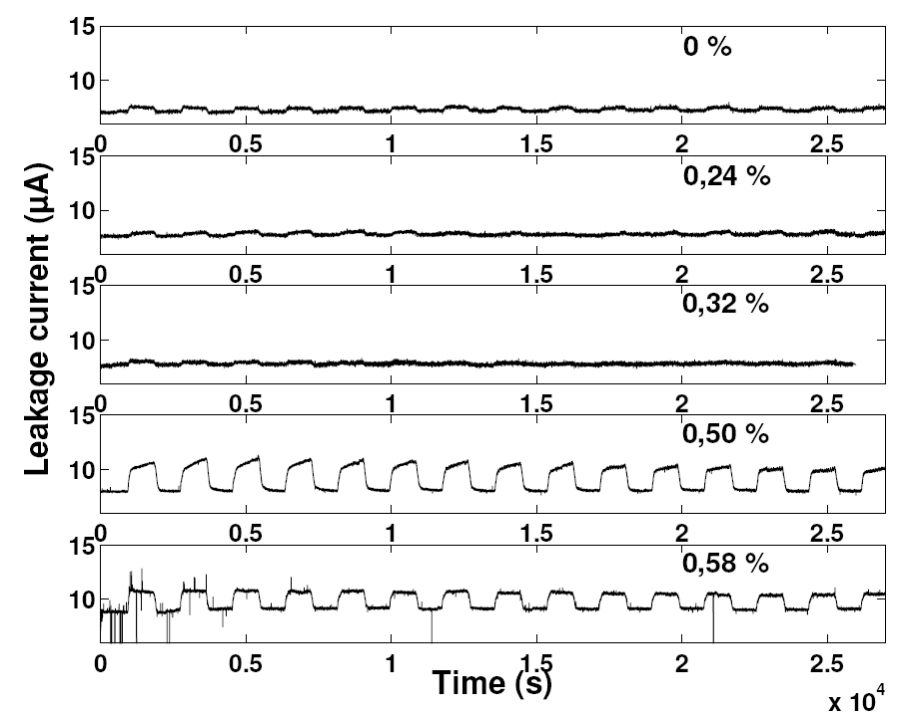

Figure 5. Evolution of the magnitude of the leakage current (RMS value of the AC current) during the electrical aging tests of THA-filled samples for initial water absorption levels ranging from 0 to 0.58 weight $\%$.

All samples display internal temperatures equal, within measurement uncertainty, to the chamber temperature, $40 \pm 2$ ${ }^{\circ} \mathrm{C}$ : we conclude that no detectable heating is associated with these leakage currents.

\subsubsection{ELECTRICAL RESPONSES OF SILICA-FILLED SMOOTH EPOXY}

Figure 6 presents the evolution of the leakage current for various initial water absorption levels in smooth silica-loaded epoxy samples ranging from 0 to 0.33 weight $\%$. We observe slight increases of the initial leakage current level with increasing water content. The current increases are mainly observed during the wet phases of the humidity cycles. The current magnitude variations are smaller than those observed for smooth THA-filled epoxy samples.

As in the case of the THA-filled epoxy samples, the internal temperature of samples having absorbed water from 0 to 0.33 weight $\%$ stay close to the temperature of the chamber, $40 \pm 2$ ${ }^{\circ} \mathrm{C}$. For larger initial water content $(\geq 0.40 \%)$ the behavior of these samples becomes different: Figure 7 displays the evolution of the leakage current for initial water contents of $0.40,0.47$ and 0.49 weight $\%$. We observe in those three cases a slow and continuous increase with time of the leakage current until a rapid increase in the current indicates a rapid deterioration of the insulating properties of the material. The test was performed over several humidity cycles for sample 1 (dry and wet phases) while samples 2 and 3 could only be tested during the first dry phase of the cycle.

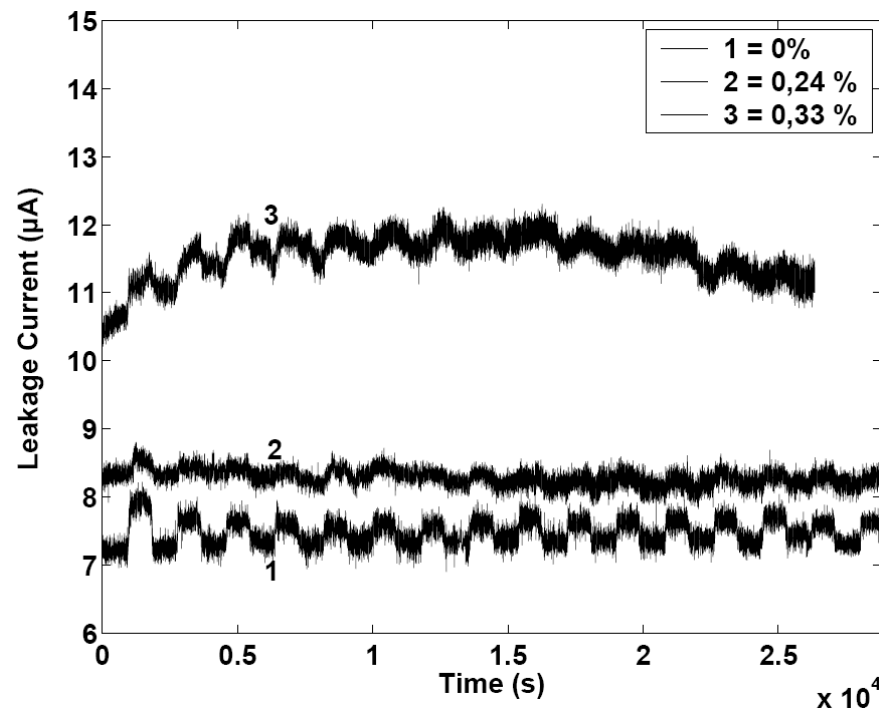

Figure 6. Evolution of the magnitude of the leakage current during electrical aging tests of silica-filled smooth epoxy samples for initial water absorption levels ranging from 0 to 0.33 sample weight $\%$.

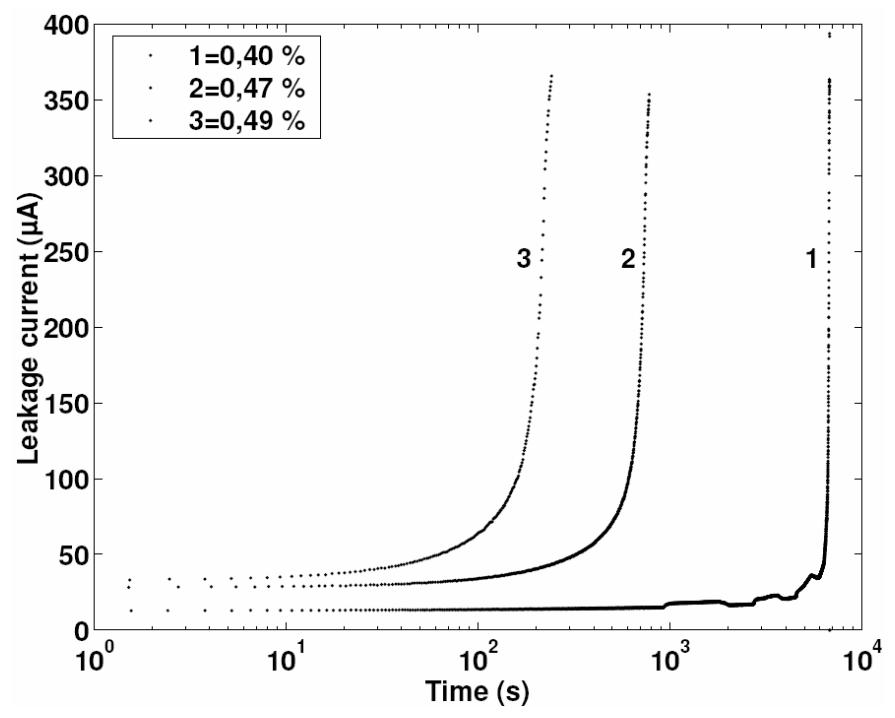

Figure 7. Magnitude of the leakage current of silica-filled smooth epoxy samples for water absorption levels from 0.40 to 0.49 weight $\%$.

Figure 8 displays the evolution of the temperature of the samples under electric stress as a function of time. We observe an increase of the temperature of the samples, sometimes exceeding the test chamber temperature.

The surface of the highly water loaded sample (initial water content 0.40 weight \%) is strongly degraded following exposure to the electric field (Figure 9): the area under the conducting elastomer was protected and does not display visible degradation, while the area exposed to the electric field appears darker and cracked.

\subsubsection{ELECTRICAL RESPONSES OF SILICA-FILLED ROUGH EPOXY}


Figure 10 displays the evolution of the leakage current as a function of time for various initial water absorption levels of rough silica filled epoxy samples. We observe that the leakage current increases with initial absorbed water levels: while the variations are moderate up to $0.33 \%$ water absorption levels, a sharp rise of the leakage current is observed above an initial water content of 0.49 weight $\%$.

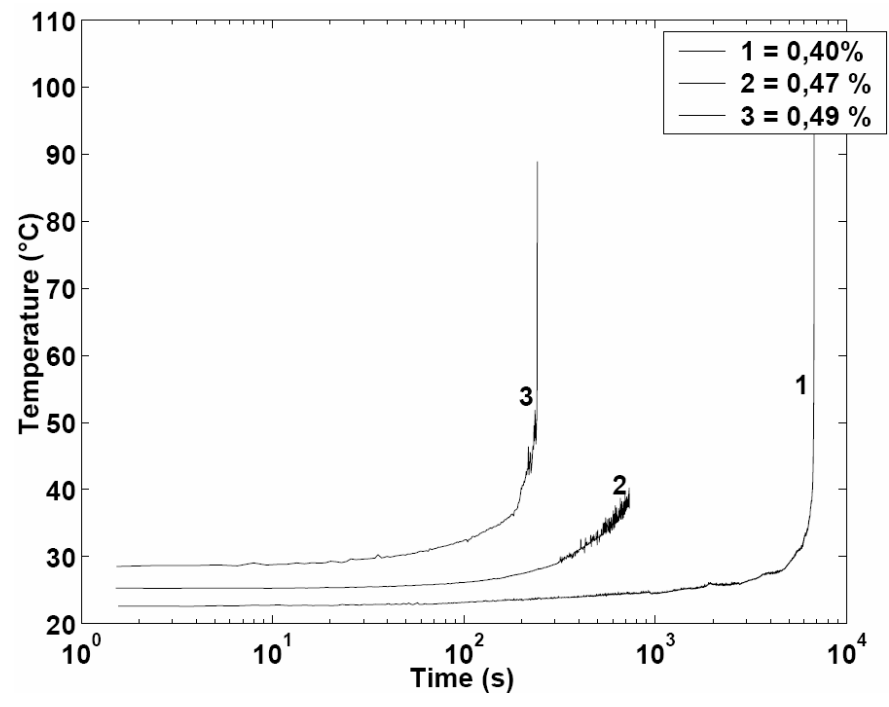

Figure 8. Evolution of the temperature of the samples with time for initial water absorption levels ranging from 0.40 to $0.49 \%$.

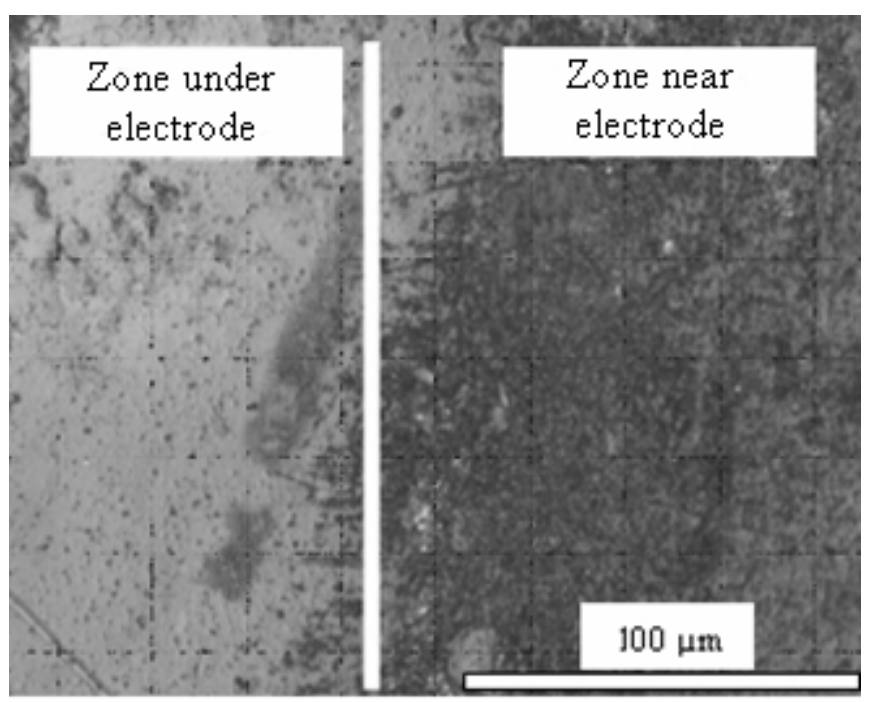

Figure 9. Optical microscope picture illustrating the degradation of the surface of a sample following electrical stress.

Figure 11 displays the evolution of the sample temperature as a function of time during electrical stress: curve 5 (characterized by an initial water absorption level of 0.66 weight \%) most obviously illustrates the fact that the temperature of the sample is well above that of the chamber, which was observed to be constant at $40 \pm 2{ }^{\circ} \mathrm{C}$.

\section{DISCUSSION}

\subsection{WATER ABSORPTION LEVELS}

The comparison of the water absorption kinetics shown in Figure 4 for the smooth and rough silica-filled epoxy samples emphasizes an important difference: the rough sample absorbs nearly twice as much water as the smooth sample. Assuming that the surface roughness affects the absorption properties of the material, one would expect some difference in behavior between the unpolished rough epoxy sample and the polished rough epoxy sample. The observation that these two samples display perfectly identical water absorption rates (Figure 4) excludes the hypothesis that surface roughness is the parameter causing this difference.

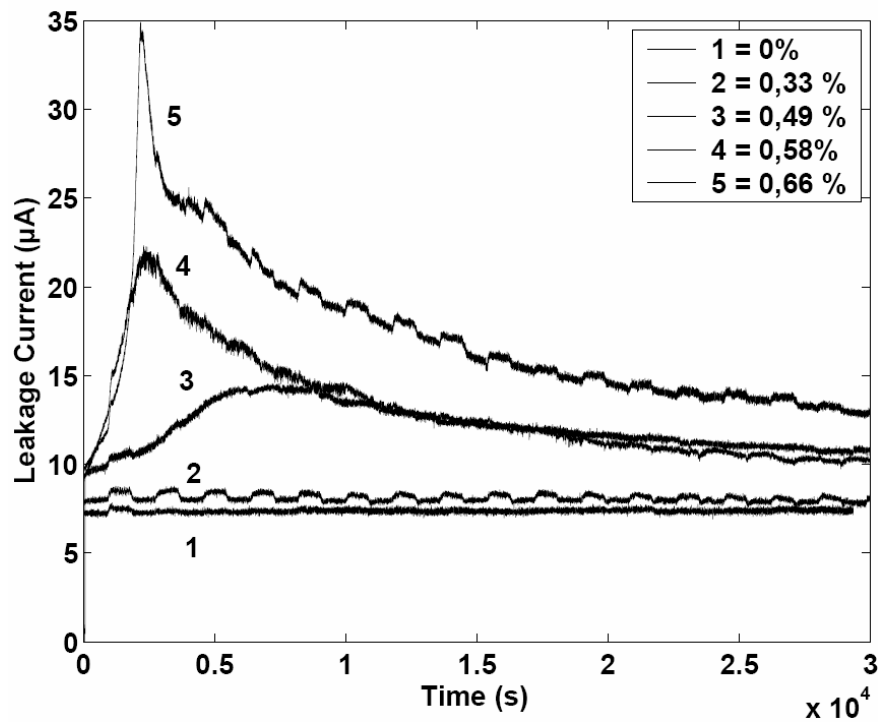

Figure 10. Leakage current for rough silica filled epoxy samples having absorbed water 0 to 0.66 weight $\%$.

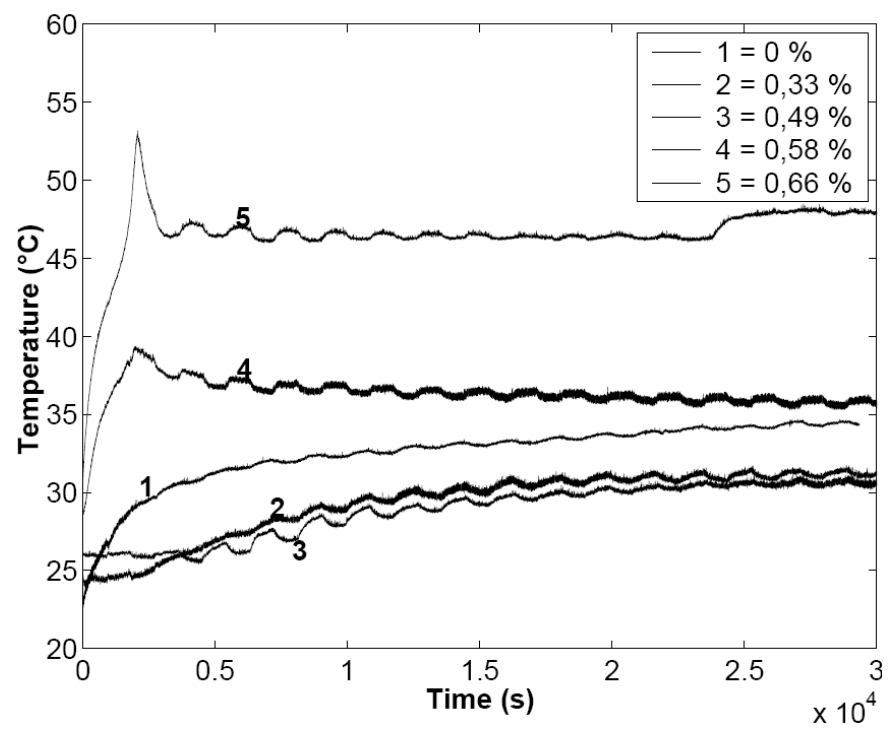

Figure 11. Temperature of the samples as a function of time for initial water absorption levels between 0 and $0.66 \%$.

Similarly, we have been unable to identify the cause of the behavior difference between the smooth silica-epoxy samples and the THA loaded samples.

Computation of the diffusion coefficients was performed assuming Fick's law [12]. The absorption rate of a liquid 
in a membrane has been described by Crank $[12,13]$. In our case the diffusion is controlled by a concentration gradient since we assume that there is no chemical reaction between the solvent and the membrane: the absorption rate is initially proportional to pt and is described by Crank - in the early stages and for a constant diffusion coefficient - as:

$$
\frac{\Delta \mathrm{m}(\mathrm{t})}{\Delta \mathrm{m}(\infty)}=2 \sqrt{\frac{\mathrm{Dt}}{\pi \mathrm{l}^{2}}}
$$

where $\Delta \mathrm{M}(\mathrm{t})$ is the mass increase due to solvent absorption at time t, $\Delta \mathrm{M}(\infty)$ is the mass increase of the solvent saturated sample, $D$ is the diffusion coefficient (in $\mathrm{m}^{2} / \mathrm{s}$ ) and 1 is the sample thickness in $\mathrm{m}$.

Computing the diffusion coefficient yields the values presented in Table 3. Table 3 shows that the unpolished rough epoxy and the polished rough epoxy samples have similar diffusion coefficients while that of the smooth silica loaded

Table 3. Diffusion coefficient measured for the various polymers under investigation in this study.

\begin{tabular}{c|c}
\hline Samples & $\begin{array}{c}\text { Diffusion coefficient } \\
\left(\mathrm{m}^{2} / \mathrm{s}\right)\end{array}$ \\
\hline Rough silica-filled \\
epoxy \\
$\begin{array}{c}\text { Rough silica-filled } \\
\text { epoxy polished } \\
\text { Smooth THA-filled } \\
\text { epoxy }\end{array}$ & $6.6610^{-12}$ \\
$\begin{array}{c}\text { Smooth silica-filled } \\
\text { epoxy }\end{array}$ & $6.6210^{-12}$ \\
\hline
\end{tabular}

epoxy is much smaller. All four samples display comparable diffusion coefficients with slightly higher values for rough and polished rough epoxy samples. These values are much larger than those found in the literature for other materials [14], but we are unaware of previous comparable studies for epoxy based materials.

\subsection{SMOOTH THA-FILLED EPOXY}

By modeling our system as a parallel resistance-capacitance circuit (RC), equations (2) and (3) provide the means for extracting the capacitive and resistive components of the current:

$$
\begin{aligned}
\mathrm{I}_{\text {resistive }} & =\mathrm{I}_{\text {total }} \times \cos (\varphi) \\
\mathrm{I}_{\text {capacitive }} & =\mathrm{I}_{\text {total }} \times \sin (\varphi)
\end{aligned}
$$

Figures 5 and 12 display the evolution of the current magnitude and the extracted capacitive and resistive components for two extreme water content cases $(0$ and 0.58 weight \% water absorption levels) as described in Equations (2) and (3). We observe a dependency of the leakage current on the initially absorbed water levels and the humidity during the electrical stress cycles.
We explain such an observation as follows. Firstly, if we assume that the observed leakage current is primarily due to water absorbed in the bulk of the material, the capacitive component being proportional to the relative permittivity of the polymer, increasing the water content would increase this component and hence the total current. For samples with a water content (curve number 2 on Figure 12), if the capacitive component is multiplied by 1.5 when passing from the dry phase to the humid phase, the resistive component is multiplied by 4 . Thus the resistive component is the most sensitive to the absorption of water. Such a result appears to diminish the hypothesis of a current flowing in the bulk of the material. The capacitive component of the leakage current is proportional to the electrical permittivity of the material (a bulk material property) while the resistive component is a combination of surface and/or bulk properties (through the conductivity). Since we have a much higher relative variation of the resistive component as compared to that of the capacitive one, our results are in favor of a predominantly surface current effect.

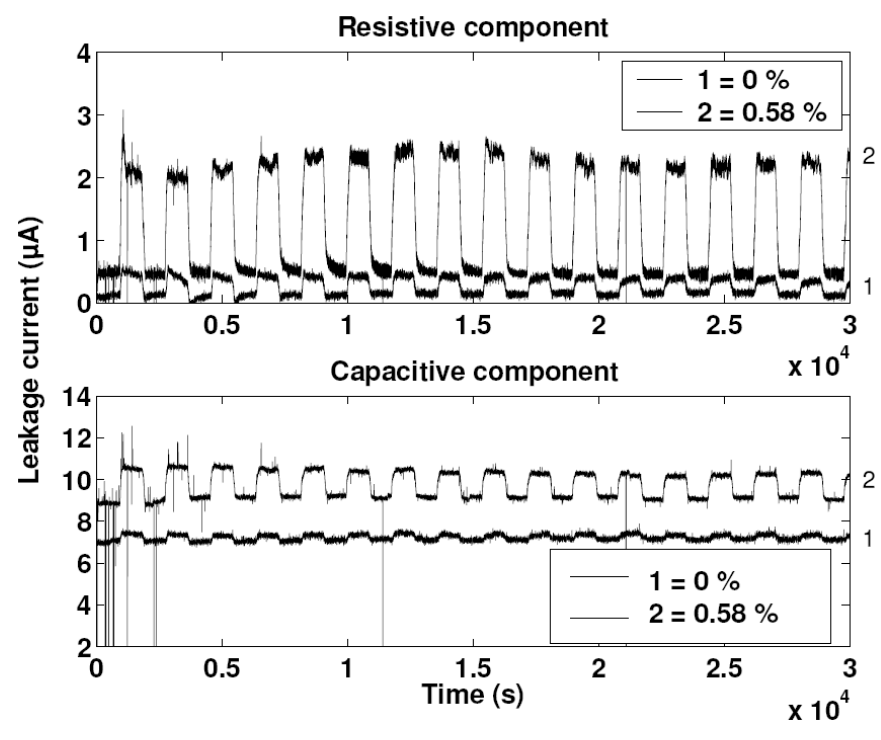

Figure 12. Evolution of the capacitive and resistive components of the leakage current for smooth THA-filled epoxy samples. The resistive component of the leakage current observed for a sample having absorbed $0 \%$ water was shifted towards the bottom of the graph by a constant value of $1 \mu \mathrm{A}$ for clarity.

In addition, the notable decrease of the resistive component of the leakage current during the dry states supports too the surface current interpretation as the surface is being dried. Indeed, during a transition from the dry to the wet states, a layer of water coats the surface and leads to an increased leakage current: such an effect is particularly observed in the resistive component of the current.

\subsection{SMOOTH SILICA-FILLED EPOXY}

Figures 13 and 14 display the evolution of the resistive and capacitive components respectively of the samples having 
absorbed 0 to $0.33 \%$ and 0.40 to 0.49 weight $\%$ of water, computed from relations (2) and (3).

As in the case of the THA filler, the current increases with increasing absorbed water levels but more significantly with humidity. If however we consider that the age resistance of the insulating material is characterized by its ability to keep the leakage current low, Figures. 7 and 14 show that the absorption of water weakens the age resistance of the material.

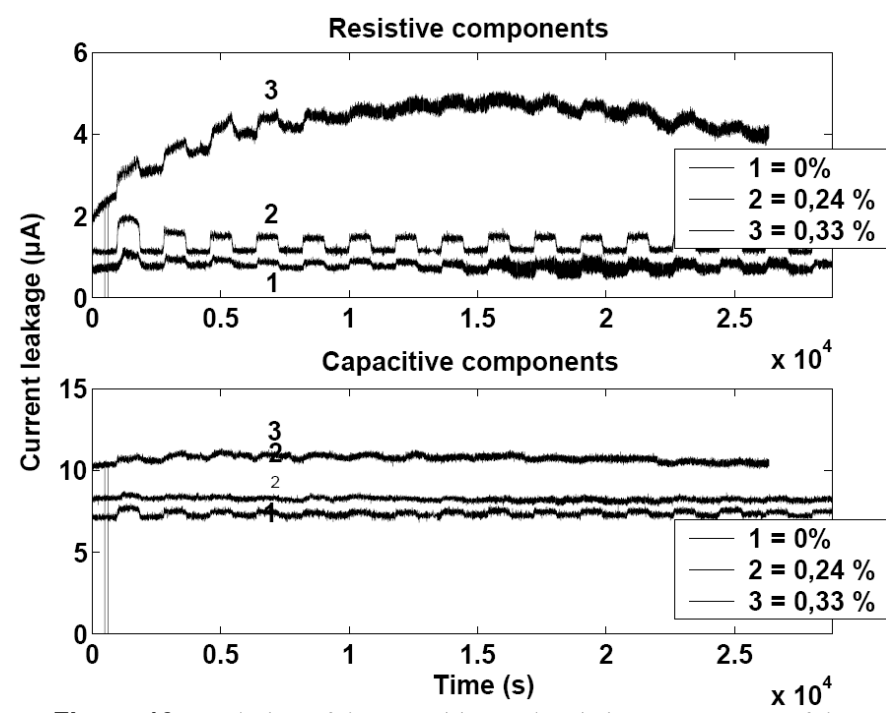

Figure 13. Evolution of the capacitive and resistive components of the leakage current for smooth silica-filled epoxy samples as a function ofinitial water absorption levels.

Humidity cycles appear not to affect the current leakage for samples with 0.40 to $0.49 \%$ water absorption levels:

For samples with $0.47 \%$ and $0.49 \%$, only the behavior during the dry phase could be observed. In these cases we also interpret the current to be flowing on the surface of the sample: were the water in the bulk of the sample to contribute to an increase of the total leakage current level, we would expect increased capacitive components associated with the increased relative permittivity of the bulk of the sample due to water.

However, in spite of the absorbed water, the capacitive component only contributes only one-fifth of the total leakage current. Replacing the THA filler with silica has degraded the electrical performances of the material, but the influence of the surface roughness is still to be investigated.

\subsection{ROUGH SILICA-FILLED EPOXY}

Referring to equations (2) and (3), we extract the capacitive and resistive components of the leakage current from the magnitude and phase measurements as shown on Figure 15. Rough silica loaded samples display an increased leakage current with initial water absorption levels. The resistive component of the leakage current closely matches the evolution of the total leakage current magnitude while the capacitive component remains nearly constant except for the sample having absorbed initially 0.66 weight $\%$ of water. In the latter case, the capacitive component slightly increases when the total leakage current magnitude reaches a maximum. We explain such an observation by stating that water absorbed in the material migrates to the surface during the aging test, hence forming a thin water layer acting as a conduction path for the leakage current. Due to Joule heating, the material heats up as seen on Figure 11.

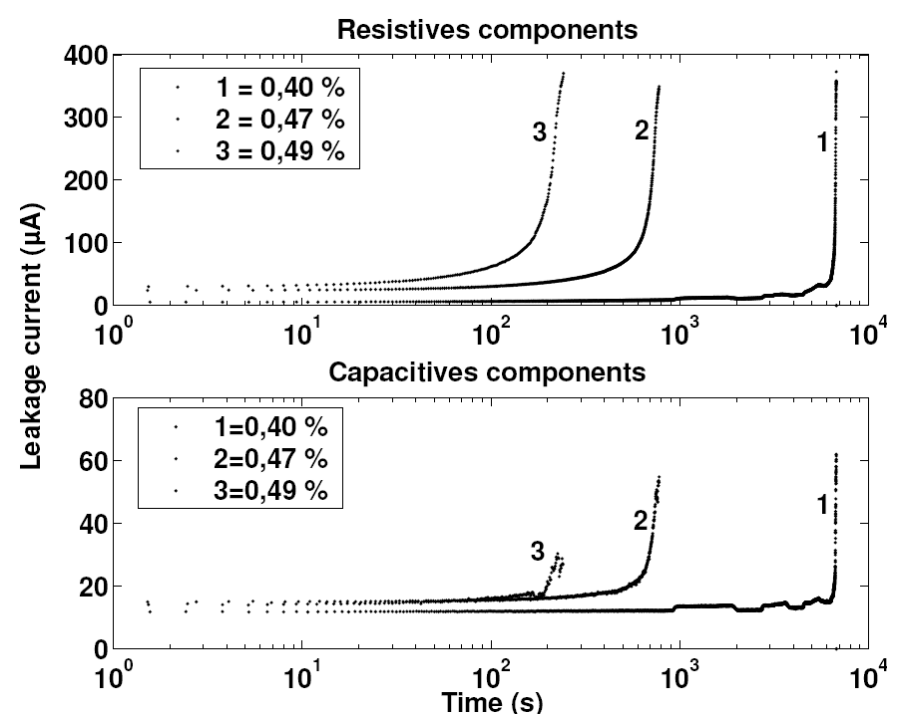

Figure 14. Capacitive and resistive components of the leakage current for initial water absorption levels ranging from 0 to 0.66 weight $\%$.

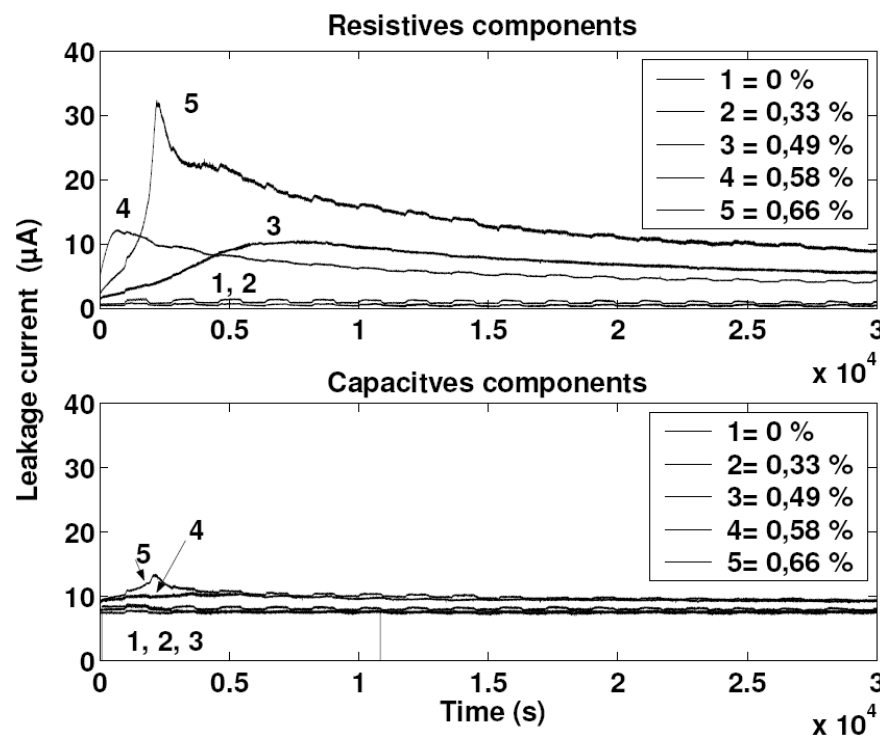

Figure 15. Capacitive and resistive components of the leakage current for initial water absorption levels ranging from 0 to 0.66 weight $\%$.

\section{CONCLUSION}

This study of the electrical behavior of epoxy insulators loaded with tri-hydrated alumina and silica as a function 
of water absorption level emphasized several characteristic behaviors of these materials:

- the water absorption rate is independent of the roughness of the sample, but is influenced by the kind of filler used,

- a high humidity level leads to an increase of the leakage current in smooth silica filled samples,

- a THA-filler greatly improves the electrical properties of the material by making it less sensitive to water content, even though the exact mechanism by which these fillers act has not been identified,

- the leakage current in the three samples under investigation here is attributed primarily to surface conduction.

\section{REFERENCES}

[1] U. Kaltenborn, P. Meier, and Y. Dirix, "Loss and recovery of hydrophobicity of novel hydrophobic epoxy resins", IEEE Conf. Electr. Insul. Dielectr. Phenomena (CEIDP), pp. 303- $306,2002$.

[2] R. Gorur, E. Cherney, R. Hackam, and T. Orbeck, "The electrical performance of polymeric insulating materials under accelerated aging in a fog chamber", IEEE Trans. Power Delivery, Vol. 3, pp.1157-64 ,1988.

[3] P. Blackmore and D. Birtwhistle, "Surface discharges on polymer insulator shed surfaces", IEEE Trans. Dielectr. Electr. Insul., Vol. 4, pp. 210-217, 1997.

[4] P. Gonon, A. Sylvestre, J. Teysseyre, and C. Prior, "Combined effects of humidity and thermal stress on the dielectric properties of epoxy-silica composites", Materials Sci. Eng. B, Vol. 83, Number 1, pp. 158-164, 2001.

[5] Y.Dirix, P. Meier, U. Kaltenborn, and V. Tilliette, "Stability of novel epoxies exposed to uv-radiation, hihg humidity and temperature", IEEE Conf. Electr. Insul. Dielectr. Phenomena (CEIDP), pp. pp.589 - 592, 2001.

[6] A. Philips, D. Childs, and H. Schneider, "Aging of non-ceramic insulator due corona from water drops", IEEE Trans. Power Delivery, Vol. 14, pp. 1081 - 1089, 1999.

[7] H.-J. Kloes and D. Koenig, "Basic investigations of the performance of droplets on electrically stressed polymer surfaces", IEEE Conf. Electr. Insul. Dielectr. Phenomena (CEIDP), pp. 374 - 377, 1997.

[8] I. Lopes, H. Shesha, Jayaram, and E. Cherney, "A study of partial discharges from water droplets on a silicone rubber insulating surface", IEEE Trans. Dielectr. Electr. Insul., Vol. 8, pp. 262 - 268, 2001.

[9] D. Williams, A. Haddad, A. Rowlands, and H. Young, "Characterization of dry bands in clean fog on polluted insulators", IEEE Trans. Dielectr. Electr. Insul., Vol. 6, pp. 724 - 731, 1999.

[10] U. Kaltenborn, J. Kindersberger, and J. Speck, "Decomposition of products of epoxy casting during the early aging period", 10th Intern. Sympos. High Voltage Eng., (ISH), pp. 269 - 272, 1997.

[11] A. Crisci, J.-P. Gosse, and V. Ollier-Dureault, "Surface-potential decay due to surface conduction", Eur. Phys. J. Appl., pp. 107-116, 1998.

[12] J. Crank, Mathematics of Diffusion, 2nd Edition, Ed. Oxford Science Publications, 1975.

[13] J. Cabanelas, S. Prolongo, B. Serrano, J. Bravo, and J. Baselga, "Water absorption in polyaminosiloxane-epoxy thermosetting polymers," J. Materials Processing Technology, Vol. 143-144, pp. 311-315, 2003.

[14] H. Zhang and R. Hackam, "Electrical surface resistance, hydrophobicity and diffusion phenomena in PVC," IEEE Trans. Dielectr. Electr. Insul., Vol. 6, pp. 73-83, 1999.
Eric Mboungou was born in Brazzaville, Congo, in 1974. He received a Master degree in Chemistry from the University of Abidjan, Ivory Coastin 1999 and a Ph.D. degree in 2006 from the University of Franche-Comte,

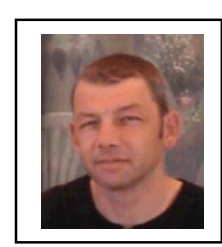
France.

Christophe Mavon was born in Besançon, France, in 1966. He received a Master degree in 1990 and a Ph.D. degree in 1996 from the University of Franche-Comte, France. Since 1996, he has worked as a research engineer at the Laboratoire de Microanalyses Nucléaires - A. Chambaudet. His research is centered on the interactions between radiations and materials notably in the field of the durability of polymers.

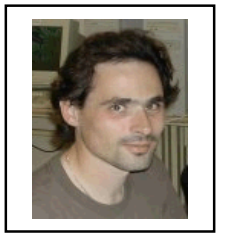

Jean-Michel Friedt was born in France in 1974. He obtained the Ph.D. degree from the University of Franche-Comté in Besancon (France) in 2000. His research activities include scanning probe microcscopy, RF surface acoustic waves and optical (bio)sensors, oscillators and radiation detection. $\mathrm{He}$ was a postdoctoral fellow with the Laboratoire de Microanalyses Nucleaires in 2004 before joining the company SENSeOR.

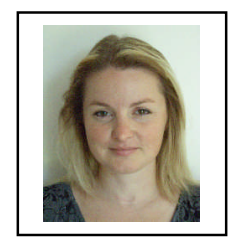

Christelle Bergeon Christelle Bergeon was born in France in 1974. She received the Ph.D. degree in 2001 from the University of Franche Comté in Besancon (France). Until 2004, she studied the interaction of radiation with polymers. Since 2005, she has specialized in the measurement of the radioactivity in water.

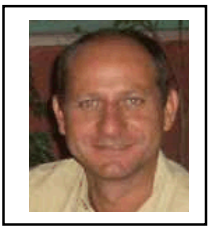

Michel Fromm was born in Colmar, France in 1962. He receivedthe Ph.D. degree in 1990 from the Université de Franche-Comté, France. After a three year position as Research Engineer in Electron Microscopy at the Faculty of Pharmacy and Medecine of Besançon he held an Assistant Professor position in Physical Chemistry at the University of Franche-Comté in 1993. In 2002 he was elected Director of the Laboratoire de Microanalyses Nucleaires-Alain Chambaudet of the same university. In 2003, he became Professor at the Université de Franche-Comté in Physical Chemistry and in the same year the laboratory became a Mix Unit of the French Atomic Agency (CEA). His current research interests are linked with the Nuclear Track Structure and Radiobiology. Since 1998 he is the secretary of the Internationnal Nuclear Track Society (INTS). 